\title{
Correction to: Resilience and Regional Dynamics
}

\author{
Hugo Pinto, Teresa Noronha, and Eric Vaz
}

\section{Correction to:}

\section{H. Pinto et al. (eds.), Resilience and Regional Dynamics, Advances in Spatial Science, https://doi.org/10.1007/978-3-319-95135-5}

This online version of this book was inadvertently published with the secondary affiliation of the editor. This has now been rectified with the primary affiliation.

\section{Editor and affiliation}

Hugo Pinto

Centre for Social Studies

University of Coimbra

Coimbra, Portugal 